\title{
Structure of $\boldsymbol{u}$ - Injective Hull in Ultra-Quasi-Pseudo Metric Space
}

\author{
Qazi Aftab Kabir ${ }^{1}$, Rizwana Jamal ${ }^{2}$, Masroor Mohammad ${ }^{3}$, Qazi Arshad Shabir ${ }^{4}$ \\ ${ }^{1,3}$ Department of Mathematics, Saifia Science College Bhopal, India \\ ${ }^{2}$ Professor and Head, Department of Mathematics, Saifia Science College Bhopal, India. \\ ${ }^{4}$ Department of Mathematics, UIT Barkatullah university Bhopal, India
}

*Corresponding Author: Qazi Aftab Kabir, Department of Mathematics, Saifia Science College Bhopal, India.

\begin{abstract}
In this paper we establish fixed point theorem for nonexpansive mappings in u-injective ultraquasi-pseudo-metric space. We generalize Agyingi [2] ultra-quasi-metric tight extension of ultra-quasimetric space to the case of nonexpansive mappings in u-injective ultra-quasi-pseudo-metric space. Our results are the extension of the results obtain by [2] and also we will generalize some crucial results about tight extensions of ultra-quasi-pseudo metric spaces.
\end{abstract}

Keywords: u-Injective hull, q-hyperconvexity, tight extension, tight span, ultra-quasi-metric space.

\section{INTRODUCTION}

The idea for injective hull of a metric space was introduced by Isbell. In [6] Isbell showed that every metric space $X$ has an injective hull $T_{X}$ which is compact if $X$ is compact. Dress [3] presented also examine the idea of the tight extension of a metric space. It is known that Dress's theory is equivalent to the theory of the injective hull of a metric space independently discussed by Isbell some years earlier. Since one of the most interesting concepts in the theory of hyperconvex metric spaces is the notion of the injective hull of a metric space [5], it is very natural to wonder about the existence of an injective hull in the field of generalized metric spaces. From that point forward a considerable measure from claiming worth of effort have been carried in regards to those hyperconvexity for nonexpansive mappings (see [ $2,4,7,9]$ ).

Kunzi and Olela Otafudu [10] presented the construction of the ultra-quasi-metrically injective hull in the category of $T_{0}$-ultra-quasi-metric spaces and contracting maps. Comparable studies have been done in the category of ultra-metric spaces and contracting maps by Bayod and Maurica [13].

In this paper we prepare the structure for u-injective hull of ultra-quasi-metrically by tight extension for ultra-quasi-pseudo-metric space for nonexpansive maps. The principle consequence of this paper is to the u-injective hull for ultra-quasi-metrically Toward tight extension for ultra-quasi-pseudometric space with nonexpansive maps. Furthermore, we examine those ultra-quasi-metrically uinjective hull from claiming an ultra-quasi-pseudo-metric space will be parallel of the q-hyperconvex hull of an ultra-quasi-pseudo-metric space.

\section{Preliminaries}

A metric space $(X, d)$ is called hyperconvex if any collection $\left\{\left(x_{i}, r_{i}\right)\right\}_{i \in I} \subset X \times[0, \infty)$ with the property that $d\left(x_{i}, r_{i}\right) \leq r_{i}+r_{i}$ for all pairs of indices $i, j \in I$, satisfies $\bigcap_{i \in I} B\left(x_{i}, r_{i}\right) \neq \emptyset$ where $B(x, r)=\{y \in X: d(x, y) \leq r\}$. With the help of zorn's lemma.

Definition 2.1. Let $X$ be set and let $u: X \times X \rightarrow[0, \infty)$ be a mapping into the set $[0, \infty)$ of nonnegative reals. Then $u$ is an ultra-quasi-pseudo-metric space on $X$ if

$$
\text { (a) } u(x, x)=0 \forall x \in X \text {, }
$$

(b) $u(x, z) \leq \max \{u(x, y), u(y, z)\}$ whenever $x, y, z \in X$ we remark here that the conjugate $u^{t}$ of $u$ where $u^{t}(x, y)=u(y, x)$ whenever $x, y \in X$ is also ultra-quasi-pseudo-metric on $X$. 
If $u$ also satisfies the condition,

(c) for any $x, y \in X, u(x, y)=0=u(y, x)$ implies that $x=y$, then $u$ is called ultra-quasi-pseudometric on $X$.

Notice that $u^{s}=\max \left\{u, u^{t}\right\}=u \vee u^{t}$ is ultrametric on $X$

Definition 2.2. A map $f:\left(X, d_{X}\right) \rightarrow\left(Y, d_{Y}\right)$ between two ultra-quasi-pseudo-metric space $\left(X, d_{X}\right)$ and $\left(Y, d_{Y}\right)$ is called nonexpansive if $d_{Y}(f(x), f(y)) \leq d_{X}(x, y)$ holds for any

$$
x, y \in X
$$

Corollary 2.3 (see [10]). Let $(X, u)$ be an ultra-quasi-pseudometric space. Consider $f: X \rightarrow[0, \infty)$ and let $x, y \in X$. Then the following are equivalent:

(a) $n(f(x), f(y)) \leq u(x, y)$;

(b) $f(x) \leq \max \{f(y), u(x, y)\}$.

Corollary 2.4 (see [10]). Let $(X, u)$ be an ultra-quasi-pseudo-metric space.

(a) Then $f:(X, u) \rightarrow([0, \infty), n)$ is a nonexpansive map if and only if

$f(x) \leq \max \{f(y), u(x, y)\}$ whenever $x, y \in X$.

(b) Then $f:(X, u) \rightarrow\left([0, \infty), n^{-1}\right)$ is a nonexpansive map if and only if

$f(x) \leq \max \{f(y), u(y, x)\}$ whenever $x, y \in X$.

\section{Q-Hyperconvex Hulls of Ultra-QuaSi-PSeudo-Metric SPaCeS}

Next we recall some basic concepts mainly from $[10,13]$ belonging to the theory of the q-hyperconvex hull of an ultra-quasi-pseudo-metric space.

Let $(X, d)$ be a ultra-quasi-pseudo-metric space. We shall say that a function pair $f=\left(f_{1}, f_{2}\right)$ on $(X, d)$ where $f_{i}: X \rightarrow[0, \infty)(i=1,2)$ is ample provided that $d(x, y) \leq \max \left\{f_{2}(x)+f_{1}(y)\right\}$ whenever $x, y \in X$.

Let us denote by $u P_{X}$ the set of all ultra-ample function pairs on ultra-quasi-pseudo-metric space $(X, d)$. For each $f, g \in P_{X}$,

$$
N(f, g)=\max \left\{\sup _{x \in X} n\left(f_{1}(x), g_{1}(x)\right), \sup _{x \in X} n\left(g_{2}(x), f_{2}(x)\right)\right\},
$$

Then $\mathrm{N}$ is an extend ultra-quasi-pseudo-metric on $P_{X}$. we recall that $\left(Q_{X}, D\right)$ is said to be the qhyperconvex hull of $(X, d)$. A ultra-quasi-pseudo-metric space $X$ is said to be q-hyperconvex if $f \in Q_{X}$ implies that there is an $x \in X$ such that $f=f_{x}$.

Definition 3.1. A quasi-pseudo-metric space $(X, d)$ is called q-hyperconvex that for each family $\left(x_{i}\right)_{i \in I}$ of points in $X$ and families $\left(r_{i}\right)_{i \in I}$ and $\left(s_{i}\right)_{i \in I}$ of nonnegative real numbers satisfying $d\left(x_{j}, x_{j}\right) \leq r_{i}+s_{j}$ whenever $i, j \in I$, the following condition holds:

$$
\bigcap_{i \in I}\left(C_{d}\left(x_{i}, r_{i}\right) \cap C_{d^{t}}\left(x_{i}, s_{i}\right)\right) \neq \emptyset
$$

\section{Injective Hull of Ultra-Quasi-Pseudo Metric Tight Extensions}

In this section we will generalize some crucial results about tight extensions of metric spaces from [5] to our injective hull in ultra-quasi-pseudo metric setting as defined below. Herrlich [7] prove that hyperconvex hull is equal to injective hull. It will be not difficult to substantiate proportionality the middle of Injectivity What's more Hyperconvexity.

Definition 4.1. [6] A metric space $(X, d)$ is said to be injective if it has the following extension property whenever $Z$ is a subspace of a metric space $Y$ and $f: Z \rightarrow X$ is nonexpansive, then $f$ has a nonexpansive extension $\bar{f}: Y \rightarrow X$. 
Definition 4.2. Let $X$ be a subspace of q-hyperconvex-ultra-quasi-pseudo-metric space $\left(Y, d_{Y}\right)$. Then $\left(Y, d_{Y}\right)$ is called tight extension of $X$ if for any ultra-quasi-pseudo-metric $\rho$ on $Y$ that satisfies $\rho \leq d_{Y}$ and agrees with $d_{Y}$ on $X \times X$, we have that $\rho=d_{Y}$.

Remark 4.3. For any $q$-hyperconvex-ultra-quasi-pseudo-metric tight extension $Y_{1}$ of $X$, any $q$-hyperconvex-ultra-quasi-pseudo metric extension $\left(Y_{2}, d\right)$ of $X$ and any nonexpansive map $\varphi: Y_{1} \rightarrow Y_{2}$ satisfying $\varphi(x)=x$ whenever $x \in X$.

It was shown in [8] that the map $e_{X}:(X, d) \rightarrow\left(Q_{X}, N\right)$ from ultra-quasi-pseudo metric space $(X, d)$ to its ultra-quasi-metrically injective hull $\left(Q_{X}, N\right)$ defined by $e_{X}(a)=f_{a}$ whenever $a \in X$ is an isometric embedding. We will proceed now with the help of (lemma 4.4) to show that $Q_{X}$ is tight extension of $e_{X}(X)$ with quasi metrically injective hull.

Lemma 4.4 (see [3], Remark 7). Let $(X, u)$ be a $u$-injective hull of ultra-quasi-pseudo-metric space. Then for any $f, g \in Q_{X}$, we have that

$$
N(f, g)=\sup \left\{d\left(x_{1}, x_{2}\right): x_{1}, x_{2} \in X, d\left(x_{1}, x_{2}\right)>f_{2}\left(x_{1}\right), d\left(x_{1}, x_{2}\right)>g_{1}\left(x_{2}\right)\right\} .
$$

Proposition 4.5 Let $(X, d)$ be $u$-injective hull of ultra-quasi-pseudo metric space and let $e_{X}: X \rightarrow Q_{X}$ be as defined above. Then $Q_{X}$ is tight extension of $e_{X}(X)$.

Proof. Let $\rho$ be $u$-injective hull of ultra-quasi-pseudo metric on $Q_{X}$ such that $\rho \leq N$ and $\rho\left(f_{x}, f_{y}\right)=$ $N\left(f_{x}, f_{y}\right)$ whenever $x, y \in X$. By lemma 3.1 and the fact that $\rho \leq N$, for any

$f, g \in Q_{X}$, we have

$$
\begin{aligned}
& N(f, g)=\sup _{x_{1}, x_{2} \in X}\left\{N\left(f_{x_{1}}, f_{x_{2}}\right): N\left(f_{x_{1}}, f_{x_{2}}\right)>\left(f_{x_{1}}, f\right), N\left(g, f_{x_{2}}\right)\right\} \\
& \leq \sup _{x_{1}, x_{2} \in X}\left\{\rho\left(f_{x_{1}}, f_{x_{2}}\right): \rho\left(f_{x_{1}}, f_{x_{2}}\right)>\rho\left(f_{x_{1}}, f\right), \rho\left(g, f_{x_{2}}\right)\right\} \leq \rho(f, g),
\end{aligned}
$$

Since $\rho\left(f_{x_{1}}, f_{x_{2}}\right) \leq \max \left\{\rho\left(f_{x_{1}}, f\right), \rho(f, g), \rho\left(g, f_{x_{2}}\right)\right\}$. Thus $\rho=N$.

\section{MAIN RESUlTS}

Theorem 5.1 Let $(X, d)$ be $u$-injective-ultra-quasi-pseudo-metric space. There exists a non-expansive retraction $f: P_{X} \rightarrow Q_{X}$, that is, a map that satisfies the following conditions:

(a) $R(f(s), f(t), f(u)) \leq R(s, t, u)$ whenever $s, t, u \in P_{X}$.

(b) $f(s) \leq s$ whenever $s \in P_{X}$. (In particular we have that $f(s)=s$ whenever $s \in Q_{X}$.)

Proof. By using Zorn's lemma. Let $(X, d)$ be $u$-injective-ultra-quasi-pseudo-metric space and let $\wp$ be the set of all maps from $P_{X}$ to $P_{X}$ satisfying conditions $(a)$ and $(b)$ in this theorem.

Order $\wp$ by

$$
\begin{gathered}
f \preccurlyeq g \\
\Leftrightarrow f(s) \leq g(s) \\
\Leftrightarrow R(f(s), f(t), f(u)) \leq R(g(s), g(t), g(u))
\end{gathered}
$$

For all $s, t, u \in P_{X}$ and $f, g \in \wp$. Then $\wp \neq \varnothing$ since the identity map belongs to $\wp$.

We have to check now that $\leqslant$ is actually a partial order. Reflexivity is obvious since every map is equal to itself. Let now $f, g, h \in \wp$ such that $f \preccurlyeq g$ and $g \preccurlyeq h$, and $h \preccurlyeq f$. Consider

$$
\begin{gathered}
f \leqslant g \\
\Rightarrow(f(s))_{1} \leq(g(s))_{1}
\end{gathered}
$$




$$
\begin{gathered}
\Rightarrow(f(s))_{2} \leq(g(s))_{2} \\
\Rightarrow R(f(s), f(t), f(u)) \leq R(g(s), g(t), g(u)) \\
g \leqslant h \\
\Rightarrow(g(s))_{1} \leq(h(s))_{1} \\
\Rightarrow(g(s))_{2} \leq(h(s))_{2} \\
\Rightarrow R(g(s), g(t), g(u)) \leq R(h(s), h(t), h(u)) .
\end{gathered}
$$

$$
\begin{gathered}
h \preccurlyeq f \\
\Rightarrow(h(s))_{1} \leq(f(s))_{1} \\
\Rightarrow(h(s))_{2} \leq(f(s))_{2} \\
\Rightarrow R(h(s), h(t), h(u)) \leq R(f(s), f(t), f(u)) .
\end{gathered}
$$

$(f(s))_{1} \leq(g(s))_{1} \leq(h(s))_{1}$ and $(g(s))_{1} \leq(h(s))_{1} \leq(f(s))_{1}$ imply that $(f(s))_{1}=(g(s))_{1}=$ $(h(s))_{1}$. In a similar manner, we have that $(f(s))_{2}=(g(s))_{2}=(h(s))_{2}$ so that we conclude that $f=g=h$.

Also $R(f(s), f(t), f(u)) \leq R(g(s), g(t), g(u)), \quad R(g(s), g(t), g(u)) \leq R(h(s), h(t), h(u)) \quad$ and $R(h(s), h(t), h(u)) \leq R(f(s), f(t), f(u))$ imply that $f=g=h$. This shows that $\leqslant$ is antisymmetric.

Suppose now that $f, g, h, i \in \wp$ such that $f \preccurlyeq g, g \preccurlyeq h$ and $h \preccurlyeq i$,

$$
\begin{gathered}
f \leqslant g \\
\Rightarrow(f(s))_{1} \leq(g(s))_{1} \\
\Rightarrow(f(s))_{2} \leq(g(s))_{2} \\
\Rightarrow R(f(s), f(t), f(u)) \leq R(g(s), g(t), g(u)) \\
g \leqslant h \\
\Rightarrow(g(s))_{1} \leq(h(s))_{1} \\
\Rightarrow(g(s))_{2} \leq(h(s))_{2} \\
\Rightarrow R(g(s), g(t), g(u)) \leq R(h(s), h(t), h(u)) . \\
h \leqslant i \\
\Rightarrow(h(s))_{1} \leq(i(s))_{1} \\
\Rightarrow(h(s))_{2} \leq(i(s))_{2} \\
\Rightarrow R(h(s), h(t), h(u)) \leq R(i(s), i(t), i(u)) .
\end{gathered}
$$

$(f(s))_{1} \leq(g(s))_{1} \leq(h(s))_{1}$ and $(g(s))_{1} \leq(h(s))_{1} \leq(i(s))_{1}$ imply that $(f(s))_{1} \leq(h(s))_{1} \leq$ $(i(s))_{1}$ by transitivity of $[0, \infty)$ as a subset of $\mathbb{R}$ with the usual ordering $\leq$. Similarly, we can show that

$$
(f(s))_{2} \leq(h(s))_{2} \leq(i(s))_{2}
$$


Also $R(f(s), f(t), f(u)) \leq R(g(s), g(t), g(u)), \quad R(g(s), g(t), g(u)) \leq R(h(s), h(t), h(u)) \quad$ and $R(h(s), h(t), h(u)) \leq R(i(s), i(t), i(u))$ imply that

$R(f(s), f(t), f(u)) \leq R(h(s), h(t), h(u)) \leq R(i(s), i(t), i(u))$ Thus $f \leqslant i$. This proves that $\leqslant$ is transitive. Therefore

$(\wp, \preccurlyeq)$ is a partially ordered set.

Next we show that every chain in $\wp$ has a lower bound. Let $\emptyset \neq \mathcal{K} \subseteq \wp$ be a chain and defines $h: P_{X} \rightarrow P_{X}$ by

$$
h(s)(x)=\left(\inf _{k \in \mathcal{K}}(k(s))_{1}(x), \inf _{k \in \mathcal{K}}(k(s))_{2}(x), \inf _{k \in \mathcal{K}}(k(s))_{3}(x)\right)
$$

whenever $x \in X$. Since $k(s) \in \wp$, we have that $h(s) \in \wp$. Indeed observe that $h(s) \leq k(s) \leq s, \forall s \in$ $P_{X}$. Thus $h(s) \leq s$ and condition (a) is satisfied. To check condition (b), $R(h(s), h(t), h(u)) \leq R(k(s), k(t), k(u)) \leq R(s, t, u)$.

Indeed

$$
R(s, t, u) \geq R(k(s), k(t), k(u))
$$

Since $k \in \wp$

$$
\begin{gathered}
\geq \sup _{x \in X}\left\{r\left((k(s))_{1}(x),(k(t))_{1}(x),(k(u))_{1}(x),\right)\right\} \\
\quad \sup _{x \in X}\left\{r\left((k(u))_{2}(x),(k(t))_{2}(x),(k(s))_{2}(x),\right)\right\} \\
\geq \sup _{x \in X} \inf _{k \in \mathcal{K}}\left\{r\left((k(s))_{1}(x),(k(t))_{1}(x),(k(u))_{1}(x),\right)\right\} \\
\vee \sup _{x \in X} \inf _{k \in \mathcal{K}}\left\{r\left((k(u))_{2}(x),(k(t))_{2}(x),(k(s))_{2}(x),\right)\right\} \\
\geq \sup _{x \in X} r\left(\inf _{k \in \mathcal{K}}(k(s))_{1}(x), \inf _{k \in \mathcal{K}}(k(t))_{1}(x), \inf _{k \in \mathcal{K}}(k(u))_{1}(x)\right) \\
\vee \sup _{x \in X} r\left(\inf _{k \in \mathcal{K}}(k(u))_{2}(x), \inf _{k \in \mathcal{K}}(k(t))_{2}(x), \inf _{k \in \mathcal{K}}(k(s))_{2}(x)\right) \\
=R(h(s), h(t), h(u)) .
\end{gathered}
$$

Thus we have that condition (b) is satisfied and since $h$ is a map from $P_{X}$ to $P_{X}$, we conclude that $h \in \wp$ and $h$ is a lower bound of the chain $\mathcal{K}$ by construction. We therefore appeal to Zorn's lemma to conclude that $\wp$ has a minimal element, say $w$, with respect to the partial order $\preccurlyeq$. To complete the proof, we show that $w(s) \in Q_{X}$ whenever $s \in Q_{X}$.

For each $x \in X$, we have that $p_{x} \circ w \wp$ and $p_{x} \circ w \preccurlyeq w$. Thus my minimality of $\mathrm{w}$, we have $p_{x} \circ w=$ $w$. It therefore follows that, for each $x \in X, p_{x}(w(s))=w(s)$ whenever $s \in P_{X}$. Thus the elements in $Q_{X}$ are defined, we conclude that $w(s) \in Q_{X}$ whenever $s \in P_{X}$.

Corollary 5.2. The subspace $[0, \infty)$ of $(\mathbb{R}, u)$ is $u$-injective.

Conclusion: In this paper we have introduced concepts of $u$-injective hull in ultra-quasi-pseudo metrically tight extension for nonexpansive mappings, also studied q-hyperconvexity in ultra-quasi metric space. We have obtained some interesting result related to nonexpansive retraction in $u$-injective ultra-quasi-pseudo metric space.

\section{REFERENCES}

[1] Agyingi, C. A., Haihambo, P. and kunzi, H. P., Endpoints in T0-quasimetric spaces, in preparation. 
[2] Agyingi, C. A, Ultra-Quasi-Metrically Tight Extensions of Ultra-Quasi-Metric Spaces, Chinese Journal of Mathematics., (2015), 646018.

[3] Aronszajn, N., and Panitchpakdi, P., Extension of uniformly continuous transformations and hyperconvex metric spaces, Pacific J. Math. 6(1956), 405- 439.

[4] Bayod, J. M. and Maurica, J. M. Ultra-metrically injective spaces, Proc. Amer. Math. Soc. 101 (1987), 571-576.

[5] Dress, Trees, tight extensions of metric spaces, and the cohomological dimension of certain groups: a note on combinatorial properties of metric spaces, Adv. in Math. 53 (1984), 321-402.

[6] Espinola, R. and Khamsi, M. A., Introduction to hyperconvex spaces, in: Handbook of metric fixed point theory, Kluwer, Dordrecht, (2001), 391-435.

[7] Herrlich H., Hyperconvex hulls of metric spaces, Topology Appl., 44(1992), 181-187.

[8] Isbell, J. R., Six theorems about injective metric spaces, Comment. Math. Helvetic. 39(1964), 65-76.

[9] Kabir, Q. A., Mohammad, M., Jamal, R and Bhardwaj, R., Unique fixed points and mappings in hyperconvex metric spaces, International journal of Mathematics and its Applications. 5(3) (2017), 171177.

[10] Kemajou, E., Kunzi, H. P and Otafudu, O. O., The Isbell-hull of a di-space, Topology Appl. 159 (2012), 2463-2475.

[11] Khamsi, M.A., and Kirk, W.A., An Introduction to Metric Spaces and Fixed Point Theory, John Wiley, New York, 2001.

[12] Kunzi, H. P., and Otafudu, O. O., q-Hyperconvexity in quasi-pseudo-metric spaces and fixed point theorems, J. Function spaces and Applications, (2012), 765903.

[13] Kunzi, H. P., and Otafudu, O. O., The ultra-quasi-metrically injective hull of a $T_{0}$-ultra-quasi-metric space, Appl. Categor. Struct. 21(2013), 651-670.

[14] Salbany, S., "Injective objects and morphisms," in Topology and Its Relation to Analysis, Algebra and Combinatorics, World Scientific, Teaneck, NJ, USA, pp. (1989), 394-409.

\section{AUTHORS' BIOGRAPHY}

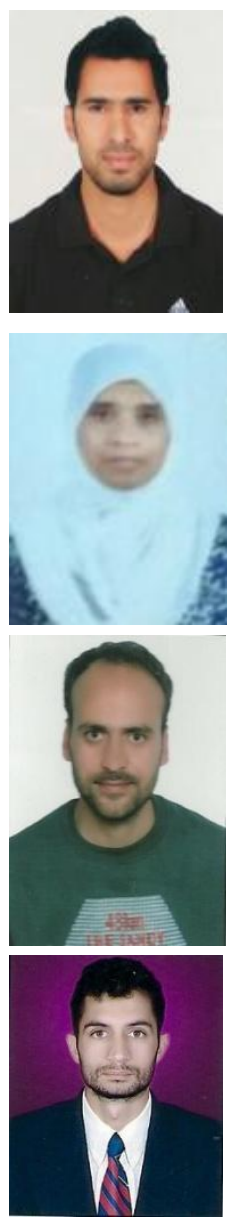

Qazi Aftab Kabir, is a research Scholar in the department of mathematics, Saifia Science College Bhopal Madhya Pradesh. He has obtained M.phil degree from Aps university rewa.

Rizwana Jamal, is a Professor in the department of Mathematics, Saifia Science College Bhopal (M.P). She has 15 years of experience in fixed point theory, topological spaces, functional analysis and metric spaces. She has published more than thirty papers in reputed journals. (IJMA, IJMAA, IJMTT, IJRDET etc.).

Masroor Mohammad, is research Scholar in the department of mathematics, Saifia Science College Bhopal Madhya Pradesh. He has obtained M.Sc. degree in mathematics from university of Kashmir.

Qazi Arshad Shabir, is a research Scholar in the department of mathematics, Barkatullah university Bhopal. He has obtained M.phil degree from UIT Barkatullah university Bhopal, India. 
Citation: Q. A. Kabir et al., "Structure of u-Injective Hull in Ultra-Quasi-Pseudo Metric Space ", International Journal of Scientific and Innovative Mathematical Research, vol. 5, no. 12, p. 40-46, 2017., http://dx.doi.org/10.20431/2347-3142.0512004

Copyright: (C) 2017 Authors. This is an open-access article distributed under the terms of the Creative Commons Attribution License, which permits unrestricted use, distribution, and reproduction in any medium, provided the original author and source are credited. 